\begin{tabular}{|c|l|}
\hline Title & Network elasticity of a model hydrogel as a function of swelling ratio : from shrinking to extreme swelling states \\
\hline Author(s) & Hoshino, Ken-ichi; Nakajima, Tasuku; Matsuda, Takahiro; Sakai, Takamasa; Gong, Jian Ping \\
\hline Citation & $\begin{array}{l}\text { Soft matter, 14(47),9693-9701 } \\
\text { https://doi.org/10.1039/C8SM01854E }\end{array}$ \\
\hline Issue Date & 2018_12-21 \\
\hline Doc URL & http://hdl.handle.net/2115/76352 \\
\hline Type & article (author version) \\
\hline File Information & Hoshino_181022_nohighlight.pdf \\
\hline
\end{tabular}

Instructions for use 


\title{
Network elasticity of a model hydrogel as a function of swelling ratio: from shrinking to extreme swelling states
}

\author{
Ken-ichi Hoshino ${ }^{a}$ Tasuku Nakajima*b,c, Takahiro Matsuda ${ }^{a}$, \\ Takamasa Sakaid, Jian Ping Gongb,c,e
}

aGraduate School of Life Science, Hokkaido University, N21W11, Kita-ku, Sapporo, Hokkaido, 001-0021, Japan

bFaculty of Advanced Life Science, Hokkaido University, N21W11, Kita-ku, Sapporo, Hokkaido, 001-0021, Japan

${ }^{c}$ Global Station for Soft Matter, Global Institution for Collaborative Research and Education, Hokkaido University, N21W11, Kita-ku, Sapporo, Hokkaido, 001-0021, Japan

dGraduate School of Engineering, The University of Tokyo, 7-3-1 Hongo, Bunkyo-ku, Tokyo, 113-0033, Japan

eInstitute for Chemical Reaction Design and Discovery (WPI-ICReDD), Hokkaido University, N21W10, Kita-ku, Sapporo, Hokkaido, 001-0021, Japan.

*tasuku@sci.hokudai.ac.jp, +81-11-706-9016 


\section{Abstract}

In this work, we intended to investigate the relationship between the swelling ratio $Q$ and Young's modulus $E$ of hydrogels from their contracted state to extreme swelling state and elucidate the underlining molecular mechanism. For this purpose, we used tetra-poly(ethylene glycol) (tetra-PEG) gel whose network parameters are well known as the polymer backbone, and succeeded in tuning the swelling of the gel by a factor of 1500 times while maintaining the topological structure of the network unchanged, using an approach combining molecular stent method and PEG dehydration method. A master curve of $Q-E$, independent of the method of obtaining $Q$, was obtained. Using the worm-like chain model, the experimentally determined master curve can be well reproduced. We also observed that the uniaxial stress-strain curve of the hydrogel can be well predicted by the worm-like chain model using the structure parameters determined from the fitting of $Q-E$ experiment curve. 


\section{Introduction}

A hydrogel is a unique soft and wet polymeric material showing a finite swelling capacity in water. From a topological point of view, the lower swelling limit of a hydrogel is its dried state, and the upper swelling limit is given by the swelling at which its network strands reach their contour length. Within this possible range of swelling, equilibrium swelling capacity and mechanical properties of a hydrogel can be widely tuned using its network and environmental conditions. Understanding the relationship between the swelling ratio and mechanical properties of a hydrogel is one of the classical problems of polymer science and numerous theoretical and experimental studies have been performed ${ }^{1-10}$. Some of the proposed swelling models of gels well reproduce the experimental behavior when the swelling ratio is sufficiently low.

While many hydrogels put to practical use (such as contact lenses) swell moderately in water, hydrogels with a large swelling capacity (so-called super-absorbent polymers) are in high demand as disposable diapers, deodorants, and stimuli-responsive actuators ${ }^{11}$. Moreover, the use of highly swollen gels is necessary for the fabrication of stiff and extremely-tough double network (DN) gels, as the brittle mechanical nature of such a highly swollen network results in sacrificial bonds to dissipate energy and toughen the DN gels ${ }^{12-15}$. Despite such recent demand and importance of highly swollen 
gels, their mechanical properties are not fully understood owing to some experimental and theoretical difficulties explained later.

Accordingly, the purpose of our study is to experimentally investigate and theoretically understand the relationship between the swelling ratio $Q$ and Young's modulus $E$ of a hydrogel, especially near its upper swelling limit. Here, we describe why swelling studies on highly swollen gels are difficult to perform experimentally. From the experimental viewpoint, to control $Q$ of the hydrogel from shrinking to highly swollen state while maintaining the network structure unchanged, polyelectrolyte (PE) gels are mostly used 16-22. As PEs have massive dissociated counterions generating large osmotic pressure, PE gels have higher swelling capacity than nonionic gels in pure water. In addition, their swelling ratio can be easily controlled using the environmental salt concentration. However, in most of the previous works, PE hydrogels were prepared via random copolymerization of ionic monomers and cross-linkers, or random post-cross-linking reactions of ionic polymers. These uncontrolled PE gel preparation methods result in inhomogeneous and defect-rich networks so that the network parameters of the resulting hydrogels are mostly unknown except the nominal $v_{\mathrm{e}}$, which is the number density of elastically effective network strands in the network estimated from the moduli of the gel. 
From the theoretical viewpoint, various theories for the swelling ratio-modulus relationship of a gel have been established after the pioneering work by Flory and Rehner ${ }^{4}$. The most classical theories including the Flory-Rehner (FR) theory assume 1) isotropic swelling, 2) each network strand being an ideal chain following Gaussian statistics, and 3) affine deformation upon swelling. Under these assumptions, the network parameters required for the model are elastically-effective chain density $v_{\mathrm{e}}$ and swelling ratio $Q$. Such simplicity of FR-like theories is compatible with the randomly-cross-linked gels whose network parameters except $v_{\mathrm{e}}$ are unknown. While FR-like theories reproduce the experimental $Q-E$ relationship to a certain extent when $Q$ is sufficiently low, they cannot predict the $E$ of highly swollen gels because the theories assume Gaussian network strands. For example, while FR-like theories predict a monotonic decrease in modulus with the increase in $Q$, the modulus of a real gel often increases with $Q$ when $Q$ is very large ${ }^{18-20}$. Such modulus upturn is caused by the non-linear strain hardening of the network strands owing to their finite extensibility, which is typically described with the inverse Langevin function or the worm-like chain model. To describe such non-linear elasticity of a gel in high-swelling regime, some swelling models considering finite extensibility of the network strands have been proposed $^{19,21-24}$. However, these models always require additional network parameters 
such as contour length of a network strand $R_{\max }$. In most cases, such additional network parameters of classical randomly-cross-linked networks cannot be determined experimentally. Thus, quantitative experimental verification of these swelling theories considering finite strand extensibility has not been performed.

To overcome these difficulties and to understand swelling ratio-modulus relationship of highly swollen hydrogels quantitatively, we designed and prepared hydrogels 1) whose network parameters are well controlled and 2) whose swelling ratio is controllable from the lower to upper swelling limits, and performed swelling experiments on them. To address 1) above, a tetra-poly(ethylene glycol) (tetra-PEG) gel with the end-link of a four-arm star polymer with a controlled molecular weight $\left(M_{\mathrm{w}} / M_{\mathrm{n}} \sim 1.05\right)$, developed by one of the authors, is chosen ${ }^{25-26}$. Given the high reaction conversion of the end-linking reaction and the negligible entanglement or elastically-ineffective loop formation ${ }^{27-30}$, the segment number $N$ of the network strand and the density of an elastically-effective network strand $v_{\mathrm{e}}$ are well defined in a tetra-PEG gel. Such a well-defined network structure of a tetra-PEG gel enables the quantitative comparison between theory and observation, which yields solutions to various unsolved problems in the polymer science field ${ }^{31-33}$. As one of the examples, systematic studies on the swelling of tetra-PEG gels in regimes of relatively low 
swelling have been already performed ${ }^{10,34}$. To address 2) above, the molecular stent method developed by other authors, in which a strong "linear" polyelectrolyte called the molecular stent is introduced to nonionic gels to increase their swelling ratio, is adopted $^{35-37}$. The high ionic osmotic pressure originating from the dissociated counterions of the trapped molecular stent greatly increases the swelling ratio of nonionic tetra-PEG gels. Swelling ratios of the gels containing a molecular stent approach their upper swelling limit with the increase in the molecular stent concentration.

We first prepared a tetra-PEG gel whose network parameters are well known, and thereafter introduced linear poly(2-acrylamido-2-methylpropanesulfonic acid sodium salt) (PNaAMPS) of varying concentrations as the polyelectrolyte stent to the tetra-PEG gel by polymerizing the NaAMPS monomer inside the tetra-PEG gel ${ }^{36-37}$. The obtained tetra-PEG gel containing PE of varying concentrations was immersed in pure water or linear PEG solutions of varying concentrations (linear PEG suppresses swelling by increasing the outside osmotic pressure) to obtain different swelling ratios $(Q)^{38}$. We subsequently measured the Young's modulus $(E)$ of the gels and investigated the experimental relationship between $Q$ and $E$. This relationship is theoretically examined by considering non-linear elasticity of the network strands using different models. 
Previous studies have shown that the mechanical properties of a swollen hydrogel are influenced by parameters such as the segment number of the network strands $(N)$ and polymer volume fractions of a gel at the state of interest $(\phi)$ and at its as-prepared state $\left(\phi_{0}\right)$. Furthermore, whether a gel is cross-linked at a semi-dilute or dilute concentration also influences its mechanical properties $6,8,10$. In this work, we prepared tetra-PEG gels with specific $N$ and $\phi_{0}$, and controlled their swelling ratios in a wide range. Thus, all the gels used in this study shared the values of $N$ and $\phi_{0}$. The gels were prepared from a pre-polymer solution of concentration $c^{*}$. Our objective is to investigate the dependence of Young's modulus $E$ on $Q\left(=\phi_{0} / \phi\right)$ of the gels cross-linked at $c^{*}$. The effects of $N$ and $\phi_{0}$ on $E$ are beyond the scope of this study. 


\section{Experiments}

\subsection{Preparation of sheet-like tetra-PEG gel}

Figure 1(a) shows the experimental scheme used to prepare samples for measurement. Tetra-PEG gels were synthesized from pre-polymer solutions via cross-end coupling as reported in the literature ${ }^{25}$. Tetraamine-terminated PEG (NOF Corporation) was first dissolved in a phosphate buffer (pH 7.4), whereas tetra-NHS-glutarate-terminated PEG (NOF Corporation) was dissolved in a citrate-phosphate buffer ( $\mathrm{pH}$ 5.8) inside a 50-mL Falcon tube. Average molecular weights of the two tetraarm-PEGs were $20 \mathrm{k}(\mathrm{g} / \mathrm{mol})$. The dispersity of each tetra-PEG macromer is 1.05 and the buffering capacity of the phosphate buffer and the citrate-phosphate buffer is $\mathrm{pH} 5.8-8.0$ and $\mathrm{pH}$ 2.6-7.0, respectively. The pre-polymer concentrations were set to their overlapping concentration of $c^{*}=40 \mathrm{mg} / \mathrm{mL}^{30}$. Subsequently, a silicone rubber frame of inner area $80 \times 80 \mathrm{~mm}^{2}$ and thickness $1.5-5$ $\mathrm{mm}$ was placed on a glass plate of size $100 \times 100 \times 3 \mathrm{~mm}^{3}$ to form a glass mold. Equal volumes of the pre-polymer solutions were mixed and injected into this glass mold by using a 10-mL plastic syringe throughout the filter membrane. Another parallel glass plate was thereafter placed on the silicone frame, and the mold composed of a pair of parallel glass plates with a silicone spacer was fixed with clips. The tetra-PEG gel 
was thereafter prepared by storing the mold for at least $12 \mathrm{~h}$ at $298 \mathrm{~K}$ until the cross-end coupling reaction was complete.

(a) Synthesis of Tetra-PEG Model Network

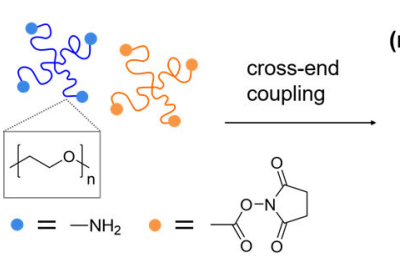

(c) Controlled Swelling in PEG Aqueous Solutions

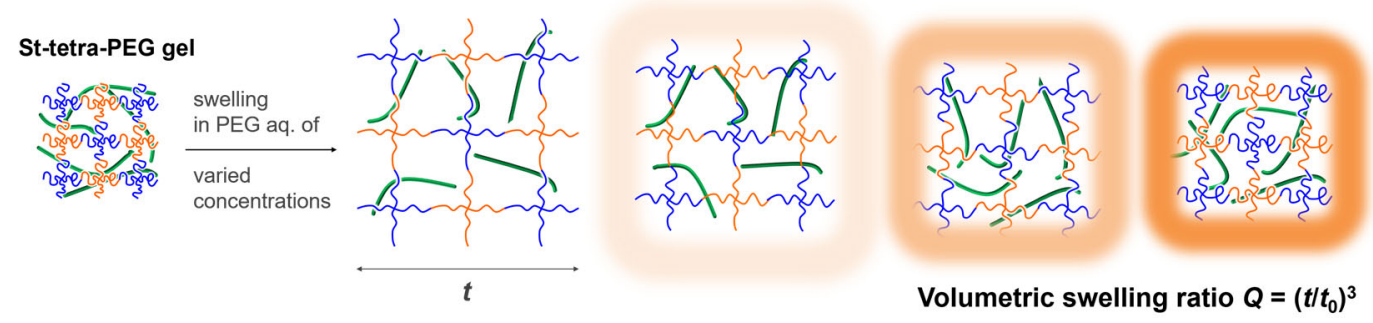

(b) Molecular Stent Introduction

Tetra-PEG gel (reference state)
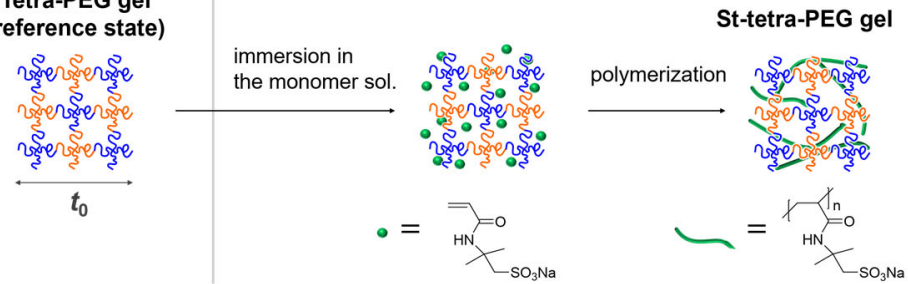

outside PEG concentration

Figure 1. Schematic illustrations of the method of obtaining a well-defined gel with a

wide range of swelling. (a) Synthesis of the tetra-PEG gels whose network parameters such as the molecular weight of the network strands are well defined, (b) introduction of a linear polyelectrolyte (molecular stent) to enhance the swelling ratio, and (c) controlled swelling of the St-tetra-PEG gels in the linear PEG aqueous solutions.

\subsection{Molecular stent method to improve swelling}

Figure 1(b) shows a schematic image of this step. Sodium 
an anionic monomer for the synthesis of the polyelectrolyte (molecular stent) for the tetra-PEG gels ${ }^{36,37}$. Accordingly, $0.2-2.5 \mathrm{M}$ of NaAMPS and $0.1 \mathrm{~mol} \%$ (relative to the monomer) of radical initiator 2-oxoglutaric acid (Wako, Pure Chemical Industries, Ltd., Tokyo, Japan) were dissolved in pure water. These solutions composed of a monomer and an initiator are called molecular stent precursor solutions. The tetra-PEG gels were immersed in these molecular stent precursor solutions for at least $24 \mathrm{~h}$. They were thereafter sandwiched by a pair of glass plates, wrapped with plastic film. Ultraviolet light (UVP lamp XX-15BLB, light intensity: $4 \mathrm{~mW} \mathrm{~cm}^{-2}$ ) at a peak wavelength of $365 \mathrm{~nm}$ was irradiated onto the glass mold from the top for at least three hours to polymerize the anionic monomers to yield the polyelectrolyte (molecular stent) in the tetra-PEG gels. The tetra-PEG gels containing the polyelectrolyte are called St-tetra-PEG gels. Notably, the synthesized PNaAMPS (molecular stent) is not covalently bonded to the tetra-PEG network backbone. Note that the introduced molecular stent itself does not contribute to the network elasticity of the tetra-PEG gel (see Figure S3 of the supplementary information). It suggests that the molecular stent is either only physically-trapped owing to entanglements or is grafted to the network backbone. Although the molecular weight of the synthesized PNaAMPS was not measured, we believe that the chains were adequately long to be mostly trapped in the gel network 
during the experiment (for at least two days), as significant oozing of the molecular stent from the gels was not observed (see Figure S1 of the supplementary information).

\subsection{PEG dehydration method to suppress swelling}

Figure 1(c) shows a schematic image of this step. The St-tetra-PEG gels were immersed in aqueous solutions of linear PEG (molecular weight: 20,000: Wako, Pure Chemical Industries, Ltd., Tokyo, Japan) of 3-33 wt.\% for at least $24 \mathrm{~h}$ until they reached new swelling equilibria. The PEG increases the osmotic pressure of the outside solution and suppresses the swelling of the network ${ }^{38}$. In this paper, this method of swelling is called the PEG dehydration method.

\subsection{Determination of volumetric swelling ratio}

As tetra-PEG gels swell isotropically owing to their isotropic network structure, the volumetric swelling ratio $Q$ of the St-tetra-PEG gels was calculated from their thickness swelling ratio $t / t_{0}$ as $Q=\left(t / t_{0}\right)^{3}$, where $t$ and $t_{0}$ are the thickness of the swollen St-tetra-PEG gel and that at its reference state (as-prepared tetra-PEG gel without molecular stent), respectively. Each value of $t$ was measured by using a laser displacement sensor LK-G3000 (Keyence Co.). Each value of $t$ (and $Q$ ) was the average 
of 10 measurements. All the experiments were performed at $298 \mathrm{~K}$.

\subsection{Indentation test}

As the fully swollen St-tetra-PEG gels were extremely fragile, their Young's moduli were determined via an indentation test using the universal mechanical testing device (Tensilon RTC-1150A: Orientec Co.) equipped with a hemisphere steel indenter. The testing velocity was $1 \mathrm{~mm} / \mathrm{min}$, which was sufficiently low for the viscous effect of the molecular stent (linear polymer) on the network modulus to be negligible and sufficiently high for the poroelastic effect on the gel to be negligible ${ }^{39}$. The Young's modulus $E$ was calculated using Hertz's contact theory, which is typically used for hard materials but is also applicable to soft materials when the depth of indentation is sufficiently small ${ }^{40,41}$. The Hertz's equation for spherical indentation is

$$
\begin{aligned}
& E=\frac{3}{4} l^{-\frac{3}{2}} f R^{-\frac{1}{2}}\left(1-v_{\mathrm{P}}^{2}\right) \\
& f=E \frac{4 R^{\frac{1}{2}}}{3\left(1-v_{\mathrm{P}}{ }^{2}\right)} l^{\frac{3}{2}}
\end{aligned}
$$

where $E, l, f, R$, and $v$ p are the Young's modulus, displacement of the indenter, force, radius of the indenter, and Poisson's ratio, respectively. $R$ in this study was $2 \mathrm{~mm}$. vP of the St-tetra-PEG gels was assumed to be 0.5 as most of hydrogels show $v_{\mathrm{P}} \cong 0.5$. $E$ was determined from the slope of the linear regime of the $f l^{\frac{3}{2}}$ curve obtained at small 
displacement $(0-0.1 \mathrm{~mm})$ based on Eq. (1b). Examples of the raw curve are shown in Figure S2 (a) in the supplementary information. The thickness of the samples for the test was set to more than $2 \mathrm{~mm}$ as the obtained slope does not depend on the thickness if it is more than $2 \mathrm{~mm}$ (see Figure $\mathrm{S} 2(\mathrm{a})$ ). We confirmed that the $E$ obtained via the indentation test was comparable to that obtained via the uniaxial stretching test (see Figure S2 (b) of the supplementary information). The values of $E$ were the average values of four measurements. All the experiments were performed at $298 \mathrm{~K}$.

\subsection{Uniaxial stretching measurement}

Uniaxial tensile tests were performed on dumbbell-shaped samples standardized as JIS K 6251-7 (12 mm in gauge length, $2 \mathrm{~mm}$ in width), using an Instron 5965 tensile tester equipped with a non-contact extensometer AVE (Instron Co.). The tensile velocity was fixed at $100 \mathrm{~mm} / \mathrm{min}$. All the experiments were performed at $298 \mathrm{~K}$.

\section{Results and Discussion}

\subsection{Estimation of the network parameters}

Given that the tetra-PEG gels have a well-defined network structure, their network parameters, which will be used for the comparison with the models, should be set from 
the preparation conditions. Here, we first calculated the values of $v_{\mathrm{e} 0}, R_{\max }$, and $R_{0}$ of the as-prepared tetra-PEG gels, where $v_{\mathrm{e} 0}, R_{\max }$, and $R_{0}$ are the number density of the elastically-effective network strands, contour length, and root-mean-square end-to-end distance of the network strands of the as-prepared tetra-PEG gels, respectively.

The $v_{\mathrm{e} 0}$ of the tetra-PEG gels was estimated using the tree-like theory for tetrafunctional networks as

$$
v_{e 0}=2 d\left(\frac{1}{2}+\left(\frac{1}{p}-\frac{3}{4}\right)^{1 / 2}\right)\left(\frac{3}{2}-\left(\frac{1}{p}-\frac{3}{4}\right)^{1 / 2}\right)^{3},
$$

where $d$ is the number density of the tetra-PEG pre-polymer in the as-prepared state, and $p$ is the conversion of the end-linking reaction of the gelation ${ }^{31}$. The $d$ of this system is given by the following equation:

$$
d=\frac{c^{*} N_{A}}{M_{w}}
$$

where $N_{A}$ is Avogadro's number and $M_{\mathrm{w}}$ is the molecular weight of the tetra-PEG macromers $(20 \mathrm{k}(\mathrm{g} / \mathrm{mol}))$. Note that the molecular weight of the network strand of the tetra-PEG gel was half that of the tetra-PEG macromers. Using a value of $p$ of 0.91 suggested by the past work ${ }^{31}, v_{\mathrm{e} 0}$ of the tetra-PEG gel can be estimated as

$$
v_{e 0}=1.64 \frac{c^{*} N_{A}}{M_{w}}
$$

For $R_{\max }$, we assume the all-trans (ttt) conformation of the PEG chains, which gives,

$$
R_{\max }=N\left(r_{1}+2 r_{2}\right) \sin \theta \quad,
$$


where $r_{1}, r_{2}$, and $\theta$ represent the length of the $\mathrm{C}-\mathrm{C}$ single bond $(0.154 \mathrm{~nm})$, length of the C-O single bond $(0.143 \mathrm{~nm})$, and half of their bond angle $\left(54.75^{\circ}\right)$, respectively. We suppose that the bond length and bond angle are not changed by elongation. For $R_{0}$, we assume that, at the as-prepared state, the tetra-PEG pre-polymers are packed with the configuration of a diamond-like lattice at their concentration $c^{*}$. Under this assumption, the distance between the two connected lattice centers $\left(=R_{0}\right)$ is given as

$$
R_{0}=\frac{\sqrt{3}}{2} d^{-\frac{1}{3}}=\frac{\sqrt{3}}{2}\left(\frac{c^{*} N_{A}}{M_{w}}\right)^{-\frac{1}{3}}
$$

By using Eqs. (2-6) and the experimental values of $M_{\mathrm{w}}$ and $c^{*}$, the estimated values of $v_{\mathrm{e} 0}, R_{0}$, and $R_{\max }$ were approximately $1.97 \times 10^{24} \mathrm{~m}^{-3}, 8.1 \mathrm{~nm}$, and $82 \mathrm{~nm}$, respectively.

\subsection{Relationship between swelling ratio and elastic modulus}

Figure 2 shows how the swelling ratio $Q$ of the tetra-PEG gels prepared at concentration $c^{*}$ was tuned by the molecular stent and PEG dehydration methods. The symbols in Figure 2(a) represent $Q$ of the swollen St-tetra-PEG gels containing the molecular stent of varying concentrations (linear PNaAMPS). $C_{\text {stent }}$ is the concentration of the feed monomer for the synthesis of the molecular stent. The addition of the molecular stent (trapped linear strong polyelectrolyte) significantly increased the value of $Q$ for the St-tetra-PEG gels up to 300, which corresponded to a $\sim 7$ times increase in 
the thickness. This extraordinary swelling was driven by the ionic osmotic pressure of the trapped counterions originating from the molecular stent. The elution of the molecular stent from the gels to the outside was confirmed to be negligible within the timescale of the experiment (see Figure $\mathrm{S} 1$ in the supporting information). The symbols in Figure 2(b) show the data for the tetra-PEG gel (without molecular stent) swollen in a series of PEG aqueous solutions with varying $C_{\text {PEG. The }} Q$ of the tetra-PEG gel gradually decreased with the increase in $C_{\mathrm{PEG}}$ owing to an increase in the osmotic pressure in the bath solution and finally reached 0.2 . While a past swelling study of the tetra-PEG gels that did not use the molecular stent method could reach $Q$ of only $2^{10,32}$, $Q$ in this study varied from 0.2 to 300 , i.e., by a factor of 1500 , through a combination of the molecular stent method and the PEG dehydration method. 


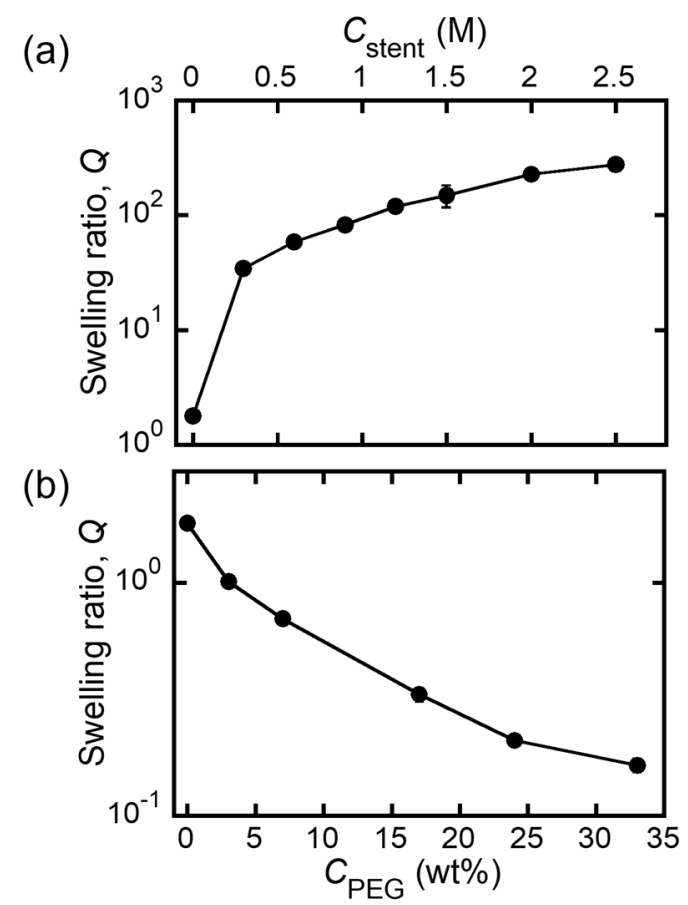

Figure 2. (a) Volumetric swelling ratio $Q$ of the St-tetra-PEG gels swollen in pure water as a function of the concentration of the feed monomer for the synthesis of the molecular stent, $C_{\text {stent. }}$ (b) $Q$ of the tetra-PEG gels swollen in the PEG aqueous solution with varied concentrations of PEG, $C_{\text {PEG. }}$

Figure 3(a) shows the $Q$ of the series of St-tetra-PEG gels containing the molecular stent with varying values of $C_{\text {stent }}$ and swollen in the PEG aqueous solutions of varying concentrations, $C_{\mathrm{PEG}}$. The $Q$ of the St-tetra-PEG gels increased with the increase in $C_{\text {stent }}$ for the same $C_{\mathrm{PEG}}$, whereas it decreased with the increase in $C_{\mathrm{PEG}}$ for the same

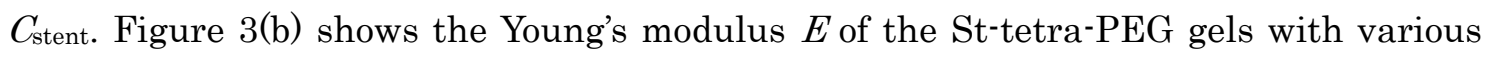
values of $C_{\text {stent }}$ and $C_{\mathrm{PEG}}$. $E$ roughly increased with an increase in $C_{\mathrm{PEG}}$ at low values of 
$C_{\text {stent }}$ and decreased with the increase in $C_{\mathrm{PEG}}$ at high $C_{\text {stent. Figure }}$ 3(c) shows the relationship between $Q$ and $E$ for St-tetra-PEG gels with varying $C_{\text {stent }}$ and $C_{\text {PEg. }}$ Interestingly, all data were on a single master curve even though these gels contained trapped molecular stent chains of varying concentrations. This result indicates that $E$ is a function of only $Q$ and does not directly depend on the concentration of the trapped molecular stent chains. This indicates that the linear polyelectrolyte topologically trapped in the gels, serving as the molecular stent, does not contribute to the measured network elasticity (this fact has also been directly confirmed as shown in Figure S3 in the Supporting information). The result thus shows that we can discuss the elasticity of the tetra-PEG network by using the correlation between $E$ and $Q$ in Figure 3(c).

According to the power-law fitting curves shown as solid lines in Figure 3(c), we classified the dependence of $E$ on $Q$ into two regimes, i.e., low-swelling (or Gaussian) ( $Q$ $<20)$ and high-swelling $(Q>20)$ regimes. The power-law exponent $\beta$ of the low-swelling regime was -0.34. In the high-swelling regime, the slope of the curve was not constant, and increased with $Q$ to finally become positive at $Q>100$.

$$
E \propto Q^{\beta} \text { where }
$$

$\beta=-0.37 \sim-1 / 3$ (low-swelling regime, $Q<20$ ),

$\beta$ increasing with $Q$ (high-swelling regime, $Q>20$ ). 
(a)

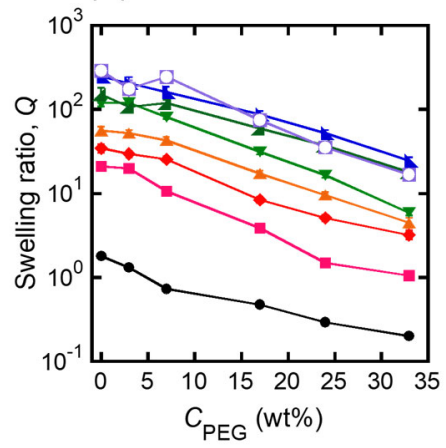

(c)

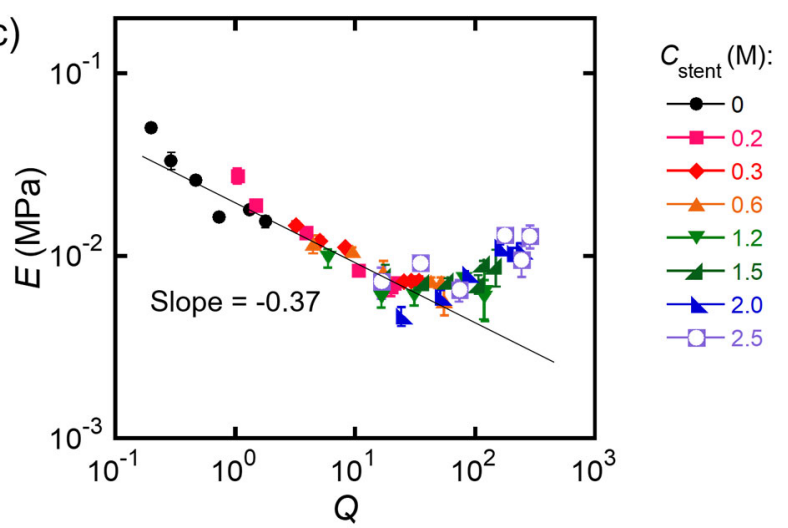

Figure 3. (a) Swelling ratio $Q$ and (b) Young's modulus $E$ of the St-tetra-PEG gels containing PNaAMPS (molecular stent) of varying concentrations equilibrated in PEG aqueous solutions of varying concentrations, $C_{\mathrm{PEG}}$. The inlet numbers represent the concentration of the feed monomer for the synthesis of the molecular stent, $C_{\text {stent. }}$ (c) The dependence of $E$ on $Q$ of the St-tetra-PEG gels with varying $C_{\text {stent }}$ and $C_{\text {PEG. The }}$ solid line represents the power-law relationship shown in Eq. (7a).

Further, we intend to rewrite Eq. (7), which is the relationship between the macroscopic properties of the gel, by using the parameters of the microscopic network strands. As the elasticity of an ideal rubbery network is the sum of elasticities of its 
network strands, the Young's modulus of a gel can be related to the elastic free energy of network strands as

$$
\frac{E}{3} \cong C v_{e l} F_{e l}^{0}
$$

where $F_{\mathrm{el}}{ }^{0}$ is the average elastic free energy of an elastically-effective network strand and $C$ is a prefactor (in the affine network model $C=1$, whereas in the phantom network model with four-functional cross-links $C=1 / 2)^{10}$. Following the previous studies on tetra-PEG gels, in this study, we adopt the phantom network model ${ }^{30} \cdot v_{\text {el }}$ is the density of the elastically-effective tetra-PEG network strands, and is inherently related to $Q$ by $v_{\mathrm{el}} \sim Q^{-1}$. Thus, $F_{\mathrm{el}}^{0}$ is proportional to $E \times Q$ as

$$
F_{e l}^{0} \propto E Q
$$

where $F_{\mathrm{el}}{ }^{0}$ should be a function of the average stretching ratio of the network strands.

Here, $\lambda$ represents the time- and ensemble-averages of the chain-stretching ratio of the network strands in the St-tetra-PEG gels compared with their relaxed states. The time average is used because the phantom network model assumes fluctuation of cross-linking points. Under the assumption of an affine deformation (not an affine network model), a microscopic $\lambda$ is equal to a macroscopic $Q^{1 / 3}$. To obtain the function shape of $F_{\mathrm{el}}{ }^{0}(\lambda)$, we plotted the $E \times Q$ of the tetra-PEG gels against $\lambda=Q^{1 / 3}$ as shown in Figure 4. The two regimes were observed in the $F_{\mathrm{el}}^{0}-\lambda$ relationship as 
$F_{e l}^{0} \propto \lambda^{\gamma}$ where

$\gamma=1.89 \sim 2 \quad$ (low-swelling regime, $\lambda<3)$,

$\gamma$ increasing with $Q$ (high-swelling regime, $3<\lambda$ ) .

(10b)

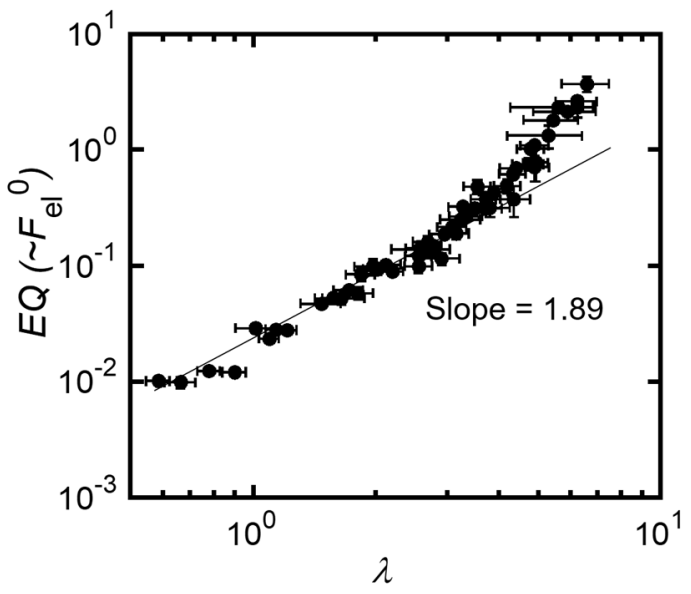

Figure 4. Dependence of $E Q$ on $\lambda\left(=Q^{1 / 3}\right)$ of the St-tetra-PEG gels with varying $C_{\text {stent }}$ and

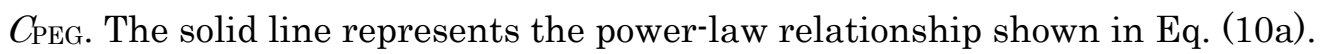

\subsection{Comparison with the elasticity models}

We now discuss the molecular origin of the above-mentioned swelling behavior of the

St-tetra-PEG gels. Recall that the St-tetra-PEG gels were synthesized from the pre-polymer solution of concentration $c^{*}$, indicating that the strands were cross-linked at dilute concentration. We first discuss the origin of Eqs. (10a) and (7a) in the low-swelling (Gaussian) regime. In this regime, network strands of the tetra-PEG gels 
can be roughly deemed a dilute solution. The behavior of this region can be understood by using prevalent models and is consistent with past studies on swollen tetra-PEG gels ${ }^{10}$. The classic chain elasticity model suggests that an isolated freely jointed polymer chain approximately behaves like a Hookean spring far from its stretching limit. Panyukov applied this single-chain elasticity theory to disordered network strands and obtained

$$
F_{e l}^{0} \cong\left(\frac{\lambda R_{0}}{R_{r e f}}\right)^{2} k_{B} T
$$

where $R_{0}$ and $R_{\text {ref }}$ are the average end-to-end distance of the network strands in an as-prepared state and the average end-to-end distance of a free chain with the same segment number at polymer concentration equivalent to that of the network of interest, respectively ${ }^{42}$. In the low-swelling regime, $R_{0}$ is roughly equal to $R_{\text {ref. }}$ Thus, in our system Eq. (11) can be written as

$$
F_{e l}^{0} \cong \lambda^{2} k_{B} T
$$

From Eqs. (9) and (12), the following equations are obtained:

$$
\begin{aligned}
& E Q \propto F_{e l}^{0}(\lambda) \propto \lambda^{2}, \\
& E \propto Q^{-1 / 3}
\end{aligned}
$$

These theoretical predictions are consistent with the experimental Eqs. (7a) and (10a).

Note that for the regime of $Q<1$, where the tetra-PEG network strands should be 
deemed a semi-dilute polymer solution, the previous studies reported different scaling laws (for example, $\beta=-0.57$ )10,34. In this work we did not observe this different scaling law, probably due to a lack of data in this regime.

Subsequently, we discuss the origin of Eqs. (7b) and (10b) in the high-swelling regime. In this regime, $F_{\mathrm{el}^{0}}$ exceeded the prediction of Eq. (7a) owing to the finite extensibility of the network strands, which was not considered by the Panyukov model and any FR-like models. Here, we adopt two single chain elasticity models considering the finite extensibility: one is the inverse Langevin chain based on a freely-jointed chain (FJC) model and the other is a worm-like chain (WLC) model. The force-extension curve of an inverse-Langevin chain can be written as

$$
f(\alpha)=\frac{k_{B} T}{b} \mathcal{L}^{-1}\left(\frac{R}{R_{\max }}\right)=\frac{k_{B} T}{b} \mathcal{L}^{-1}(\alpha)
$$

where $\mathcal{L}^{-1}, f, b, R$, and $R_{\max }$ are the inverse Langevin function, mean force, Kuhn length, mean end-to-end distance, and contour length, respectively, and $\alpha$ is $R / R_{\max }$. Eq. (14) can be simplified by using Padé approximation ${ }^{43}$ as

$$
f(\alpha)=\frac{k_{B} T}{b} \frac{\alpha\left(3-\alpha^{2}\right)}{1-\alpha^{2}}
$$

The elastic free energy of a single inverse-Langevin chain, $F_{\mathrm{el}^{0}}(\alpha)$, is given by the integral of a force-extension curve (Eq. (15)),

$$
F_{e l}^{0}(\alpha)=R_{\max } \int_{0}^{\alpha} f(\alpha) \mathrm{d} \alpha=\frac{k_{B} T R_{\max }}{b}\left(\frac{\alpha^{2}}{2}-\ln \left(\left|\alpha^{2}-1\right|\right)\right) .
$$


The reason for multiplying $R_{\max }$ is to convert the length ratio $\alpha(\mathrm{m} / \mathrm{m})$ into displacement $R(\mathrm{~m})$ to calculate the energy $(\mathrm{N} \cdot \mathrm{m})$. The Young's modulus of the tetra-PEG gel based on the inverse Langevin model is given by substituting Eq. (16) into Eq. (8) as

$$
E(\alpha)=\frac{3}{2} v_{e} F_{e l}^{0}=\frac{3 v_{e_{0}}}{2 Q} F_{e l}^{0}=\frac{3 v_{e_{0}} k_{B} T R_{\max }}{2 Q b}\left(\frac{\alpha^{2}}{2}-\ln \left|\alpha^{2}-1\right|\right),
$$

or by using $\lambda=\frac{R}{R_{0}}=Q^{\frac{1}{3}}$ as

$$
E(\lambda)=\frac{3 v_{e_{0}} k_{B} T R_{\max }}{2 Q b}\left(\frac{\left(\frac{\lambda R_{0}}{R_{\max }}\right)^{2}}{2}-\ln \left|\left(\frac{\lambda R_{0}}{R_{\max }}\right)^{2}-1\right|\right) .
$$

Note that we adopt the phantom network model ( $C=1 / 2$ in Eq.(8)).

Similarly, the force-extension curve of a WLC chain can be approximately written as

$$
f(\alpha) \cong \frac{k_{B} T}{l_{p}}\left\{\frac{1}{4}\left(1-\frac{R}{R_{\max }}\right)^{-2}-\frac{1}{4}+\frac{R}{R_{\max }}\right\}=\frac{k_{B} T}{l_{p}}\left\{\frac{1}{4}(1-\alpha)^{-2}-\frac{1}{4}+\alpha\right\},
$$

where $l_{\mathrm{p}}=b / 2$ is the persistence length ${ }^{44}$. Thus, $F_{e l}^{0}(\alpha)$ is given by

$$
F_{e l}^{0}(\alpha)=R_{\max } \int_{0}^{\alpha} f(\alpha) \mathrm{d} \alpha=\frac{k_{B} T R_{\max }}{4 l_{p}}\left(2 \alpha^{2}-\alpha-(\alpha-1)^{-1}-1\right)
$$

The Young's modulus of the tetra-PEG gel based on the WLC model is given by substituting Eq. (19) into Eq. (8) as

$$
E(\alpha)=\frac{3}{2} v_{e} F_{e l}^{0}=\frac{3 v_{e_{0}}}{2 Q} F_{e l}^{0}=\frac{3 v_{e_{0}} k_{B} T R_{\max }}{8 Q l_{p}}\left(2 \alpha^{2}-\alpha-(\alpha-1)^{-1}-1\right),(20 \mathrm{a})
$$

or by using $\lambda=\frac{R}{R_{0}}=Q^{\frac{1}{3}}$ as

$$
E(\lambda)=\frac{3 v_{e_{0}} k_{B} T R_{\max }}{8 Q l_{p}}\left(2\left(\frac{\lambda R_{0}}{R_{\max }}\right)^{2}-\frac{\lambda R_{0}}{R_{\max }}-\left(\frac{\lambda R_{0}}{R_{\max }}-1\right)^{-1}-1\right) .
$$

Among the variables in the equations, $Q, \lambda$, and $T$ are experimental parameters, $v_{\mathrm{e} 0}, R_{0}$, and $R_{\max }$ can be estimated from the preparation conditions as described before, and the 
literature value can be used for $b(0.76 \mathrm{~nm})$ and $l_{\mathrm{p}}=b / 2(0.38 \mathrm{~nm})^{45,46}$.

Based on Eqs. (17) and (20), the experimental dependence of $E$ of the tetra-PEG gels on $Q$ was fitted by the FJC and WLC models. Figure 5(a, b) shows the FJC and WLC fitting curves of $E$ versus $Q$ of the tetra-PEG gels, respectively. Two fitting curves are shown in each graph where 1) there was no fitting parameter (literature or experimental values were used for all variables), or 2) $R_{0}$ and $R_{\max }$ were treated as fitting parameters. In the setting 1), the theoretical moduli (blue dotted line in the figure) are slightly lower than the experimental values in both FJC and WLC models, and the modulus upturn at high $Q$ was not reproduced. Thus, some parameters must be modified. In the setting 2), the theoretical curves of both WLC and FJC models (purple solid line) are consistent with the experimental moduli at low-swelling regime. Moreover, both models qualitatively reproduce the upturn in the modulus at high-swelling regime. In terms of curve shapes, however, the WLC model well reproduced the experimental results whereas the FJC model predicted a too-sharp modulus upturn. Thus, we conclude that the WLC model is better than the FJC model to describe the mechanical behavior of the PEG network. The WLC theoretical curve satisfactorily reproduced the experimental values. The best WLC fitting value of $R_{0}$ was $8.9 \mathrm{~nm}$, sufficiently close to the theoretical value $(8.1 \mathrm{~nm})$. In contrast, the value of the 
fitted $R_{\max }$ was $63 \mathrm{~nm}$, which is very different from the estimated $R_{\max }(82 \mathrm{~nm}$ ). Two possible reasons for the discrepancy between the estimated $R_{\max }(82 \mathrm{~nm})$ and the fitted $R_{\max }(63 \mathrm{~nm})$ are discussed below. One possibility is the characteristic conformation of PEG in water. Although all-trans (ttt) conformation, which is the precondition for the calculation of $R_{\max }$, is the most stable conformation of PEG chains in vacuum, trans-trans-gauche (ttg) conformation is more stable in water because two neighboring oxygen atoms in PEG and two hydrogen atoms of a water molecule form hydrogen bonds ${ }^{46,47}$. If we assume the ttg conformation of the tetra-PEG network strands, the theoretical value of their contour lengths $R_{\max }$ is approximately $64 \mathrm{~nm}$, sufficiently close to the fitting value $(63 \mathrm{~nm})$. Another possibility is the contribution of the contour length distribution of the network strands. Although the tetra-PEG macromers are narrow distribution polymers showing $M_{\mathrm{w}} / M_{\mathrm{n}}$ of 1.05 , they have narrow but unignorable molecular weight distributions. The relatively short network strands in the tetra-PEG network should have smaller contour length than the (averaged) $R_{\max }$. When the network is highly swollen, the shorter strands should be more stretched and should sustain higher force than the longer strands. Thus, the mechanical properties of gels near their swelling limit should be governed mainly by the shorter strands. The $R_{\max }$ obtained via the fitting may reflect the contour length of the relatively short chains in 
the tetra-PEG network. A similar discussion could be applied to the network strands having the same contour length but with different end-to-end distances. The strands with longer initial end-to-end distance might reach their stretching limit faster and sustain higher force than the strands with shorter initial end-to-end distance.

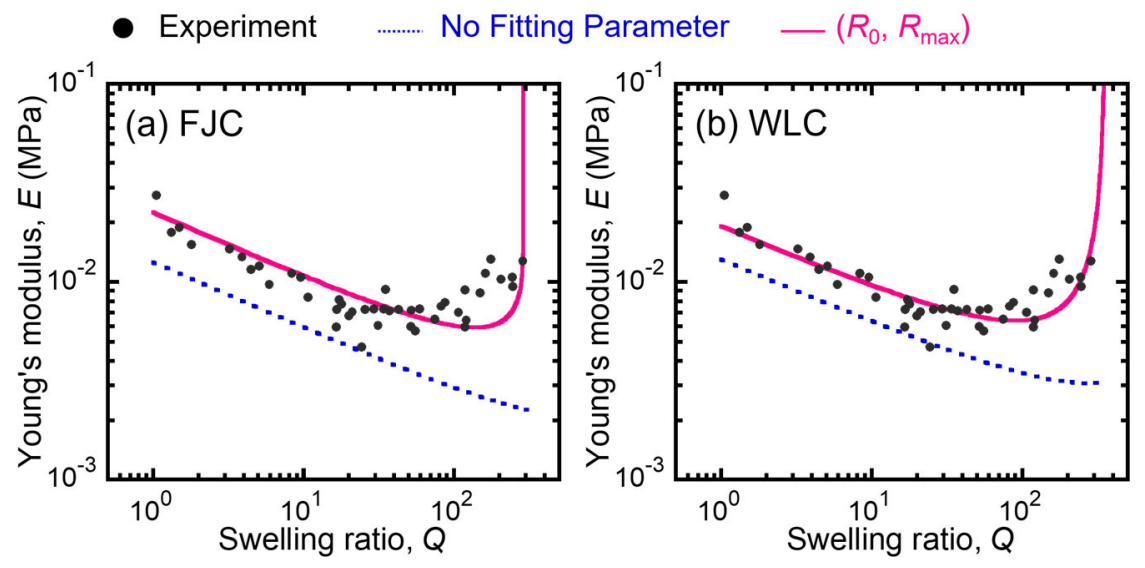

Figure 5. Results of fitting to the $E-Q$ relationship of the tetra-PEG gels; (a) FJC model fittings and (b) WLC model fittings. The dotted lines represent the curves calculated without using the fitting parameters (estimated or literature values are used for all variables), whereas the solid lines represent the best fitting curves calculated using $R_{0}$ and $R_{\max }$ as the fitting parameters. The best fitting parameters for the FJC model are $R_{0}=10.2 \mathrm{~nm}$ and $R_{\max }=67.7 \mathrm{~nm}$ whereas those for the WLC model are $R_{0}=8.9 \mathrm{~nm}$ and $R_{\max }=63.1 \mathrm{~nm}$. 


\subsection{Expansion of the model to uniaxial stretching}

By integrating $F_{\mathrm{el}^{0}}$ over the entire network, the total elastic energy of the network, $W_{\text {net, }}$ can be calculated as a function of $\lambda$. We expand our theory to the prediction of the results of the uniaxial stretching of the tetra-PEG gel. A (modified) eight-chain ArrudaBoyce model was used to theoretically consider the stretching experiment ${ }^{48}$. In the model, a polymer network was represented as the sum of cubic units, where eight network strands with the segment number connected its center to the corners. The merit of the eight-chain model is that the stretching ratio of all the chains is identical regardless of the type of deformation. The elastic energy density stored in an eight-chain model gel becomes

$$
W_{\text {net }}=C v_{e} F_{e l}^{0}\left(\lambda_{\text {chain }}\right)=C \frac{v_{e_{0}}}{Q} F_{e l}^{0}\left(\lambda_{\text {chain }}\right)
$$

For the uniaxial stretching of incompressible materials including tetra-PEG gels along the $x$-axis, the engineering tensile stress is $\sigma_{x}$, and $\sigma_{y}=\sigma_{z}=0$. The deformation ratio of each axis $\lambda_{i}(\dot{I}=x, y, z)$ should be

$$
\lambda_{x}=\lambda_{y}{ }^{-2}=\lambda_{z}{ }^{-2} \quad,
$$

and the deformation ratio of each chain in the system is

$$
\lambda_{\text {chain }}=\sqrt{\frac{\lambda_{x}^{2}+2 \lambda_{x}{ }^{-1}}{3}} .
$$

To obtain the uniaxial stress-deformation ratio relationship, Eq. (21) can be 
differentiated with $\lambda_{\mathrm{x}}$ as

$$
\sigma_{x}=\frac{\partial W_{n e t}}{\partial \lambda_{x}}
$$

Note that the eight-chain model does not necessarily assume eight-branched cross-linked points. The model simply dictates the relationship between the macroscopic deformation of the network and the microscopic deformation of its network strands.

Here, we calculated $F_{\mathrm{el}} 0(\lambda)$ based on the WLC model using Eqs. (16) and (21) with the same $R_{0}(8.9 \mathrm{~nm})$ and $R_{\max }(63 \mathrm{~nm})$, and substitute $F_{\mathrm{el}}{ }^{0}(\lambda)$ into Eq. $(21)$ to obtain $W_{\text {net. }}$ Thus, the obtained theoretical curve shown in the figure is not the fitted curve. Figure 6 shows the experimental stress-deformation ratio curve of the as-prepared tetra-PEG gel $(Q=1)$ and the theoretical curve predicted using Eq. (24). The stresses were normalized by their Young's moduli $E$. For comparison, the curve calculated with the neo-Hookean (NH) model

$$
\sigma_{x}=\frac{E}{3}\left(\lambda_{x}-\lambda_{x}{ }^{-2}\right)
$$

is also shown ${ }^{49}$. The experimental and calculated curves overlapped almost completely. In particular, the theoretical curve satisfactorily mirrors the strain hardening, which was not considered in the NH model. The maximum experimental $\lambda=Q^{1 / 3}$ of the swelling test is 6.6 , which corresponds to $\lambda_{\mathrm{x}}=11.4$. It indicates that the calculated stresses shown in the figure are not the extrapolated values but are supported by the swelling 
experiments.

The reproduction of the experimental uniaxial stretching results by our model suggests that $W_{\text {net }}$ calculated from the swelling experiment is generally applicable to the prediction of the uniaxial stress-strain behavior. This result strongly suggests the validity of the obtained strain energy-density function $\left(W_{\text {net }}\right)$ of the gels through swelling experiments as a kind of triaxial stretching. We believe that our method has the potential to complement conventional uniaxial and biaxial stretching experiments to determine $W_{\text {net }}$ of various gels and elastomers.

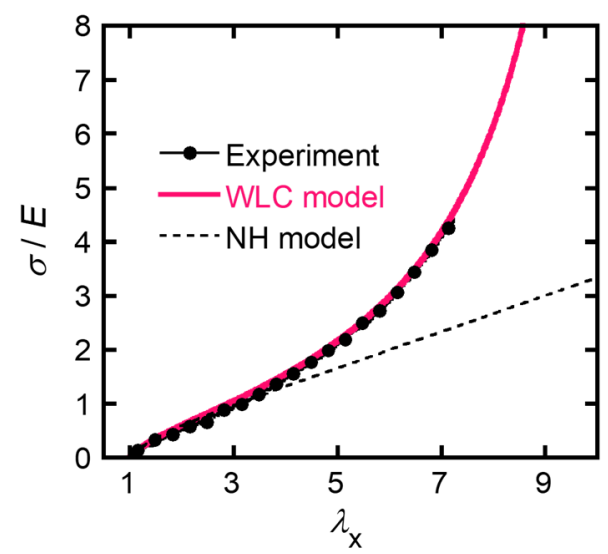

Figure 6. Uniaxial normalized stress-deformation ratio curves of the as-prepared tetra-PEG gel determined via an experiment and calculated using the WLC model (Eqs. $(19,21,24))$. The curve calculated based on NH model (Eq. (25)) is also shown for comparison. 


\section{Conclusions}

We experimentally determined the moduli of tetra-PEG hydrogels as a function of their swelling ratio up to a high-swelling regime. To the best of our knowledge, this is the first study to elucidate the swelling ratio-modulus relationship of the model networks up to their upper swelling limits. In addition, we theoretically explained the obtained swelling ratio-modulus relationship by adopting non-linearly elastic network chains with reasonable fitting parameters. It is suggested that the WLC model should be used for describing the non-linear elasticity of PEG chains instead of the FJC (inverse Langevin) model, which has been typically used for swelling models.

In terms of applications of gels, this study provides a guide for the design of hydrogel materials with a certain modulus and water content, especially at high-swelling regimes. Such fully swollen hydrogels are directly used as SAPs and are useful for the fabrication of tough DN hydrogels. Based on the elasticity model established in this study, mechanical properties of a highly-swollen gel will be able to be controlled precisely. According to previous research, mechanical behavior of a DN hydrogel at small deformation is mainly determined by the highly-swollen $1^{\text {st }}$ network structure ${ }^{20,37}$. Thus, this work will also enable controlled mechanical behavior of DN hydrogels by controlling the $1^{\text {st }}$ network structure. Such study not only provides DN hydrogels having 
targeted properties for specific medical and industrial applications, but also leads to further understanding of the toughening mechanism of DN hydrogels.

\section{Conflicts of interest}

There are no conflicts to declare.

\section{Acknowledgments}

This research was supported by JSPS KAKENHI Grant Numbers JP17H04891 and JP17H06144, and by ImPACT Program of Council for Science, Technology and Innovation (Cabinet Office, Government of Japan). Institute for Chemical Reaction Design and Discovery (ICRD) was established by World Premier International Research Initiative (WPI), MEXT, Japan. Authors thank Dr. Daniel R. King (Hokkaido University) for his help of English editing and Dr. Takuya Katashima (RIKEN, Japan) for his meaningful comments. 
1953, p.458-470, p.577-579.

3 M. Rubinstein and R. H. Colby, Polymer Physics, Oxford University Press,

Oxford, 2003, p.140-146.

$4 \quad$ P. J. Flory and J.Rehner, J. Chem. Phys., 1943, 11, 521

$5 \quad$ J. Kovac, Macromolecules, 1978, 11, 362

6 S. P. Obukhov, M. Rubinstein and R. H. Colby, Macromolecules, 1994, 27, 3191

$7 \quad$ T. Tanaka, Phys. Rev. Lett., 1978, 40, 820

8 K. Urayama and S. Kohjiya, J. Chem. Phys., 1996, 104, 3352

9 K. Urayama, T. Kawamura and S. Kohjiya, J. Chem. Phys., 1996, 105, 4833

10 T. Sakai, M. Kurakazu, Y. Akagi, M. Shibayama and U.-I. Chung, Soft Matter, $2012,8,2730$

11 J. Kollár, M. Mrlík, D. Moravčíková, Z. Kroneková, T. Liptaj, I. Lacík and J. Mosnáček, Macromolecules, 2016, 49, 4047

12 J. P. Gong, Y. Katsuyama, T. Kurokawa and Y. Osada, Adv. Mater, 2003, 15, 1155

13 H. R. Brown, Macromolecules, 2007, 40, 3815

14 Y. Tanaka, Europhys. Lett., 2007, 78, 56005

15 Y. Tanaka, Y. Kawauchi, T. Kurokawa, H. Furukawa, T. Okajima and J. P. Gong, 
Macromol. Rapid Commun., 2008, 29, 1514

16 R. Skouri, F. Schosseler, J. P. Munch and S. J. Candau, Macromolecules, 1995,

28,197

17 G. Nisato, F. Schosseler and S. J. Candau, Polym. Gels Networks, 1996, 4, 481

18 F. Horkay, I. Tasaki and P. J. Basser, Biomacromolecules, 2001, 2, 195

19 N. Gundogan, D. Melekaslan and O. Okay, Macromolecules, 2002, 35, 5616.

20 H. Itagaki, T. Kurokawa, H. Furukawa, T. Nakajima, Y. Katsumoto and J. P.

Gong, Macromolecules, 2010, 43, 9495

21 A. Katchalsky, S. Lifson and H. Exsenberg, J. Polym. Sci., 1951, 7, 571

22 A. Katchalsky and I. Michaeli, J. Polym. Sci., 1955, 15, 69

23 M. Rubinstein, R. H. Colby, A. V. Dobrynin and J.-F. Joanny, Macromolecules,

$1996,29,398$

24 Y. D. Zaroslov, O. E. Philippova, and A. R. Khokhlov, Macromolecules, 1999, 32, 1508

25 T. Sakai, T. Matsunaga, Y. Yamamoto, C. Ito, R. Yoshida, S. Suzuki, N. Sasaki, M. Shibayama and U.-I. Chung, Macromolecules, 2008, 41, 5379

26 T. Sakai, React. Funct. Polym., 2013, 73, 898

27 T. Matsunaga, T. Sakai, Y. Akagi, U.-I. Chung and M. Shibayama, 
Macromolecules, 2009, 42, 1344

28 Y. Akagi, T. Katashima, Y. Katsumoto, K. Fujii, T. Matsunaga, U.-I. Chung, M.

Shibayama and T. Sakai, Macromolecules, 2011, 44, 5817

29 F. Lange, K. Schwenke, M. Kurakazu, Y. Akagi, U.-I. Chung, M. Lang, J. U.

Sommer, T. Sakai and K. Saalwächter, Macromolecules, 2011, 44, 9666

30 Y. Akagi, J. P. Gong, U.-I. Chung and T. Sakai, Macromolecules, 2013, 46, 1035

31 Y. Akagi, T. Katashima, H. Sakurai, U.-I. Chung and T. Sakai, RSC Advances, $2013,3,13251$

32 X. Li, K. Khairulina, U. Chung and T. Sakai, Macromolecules, 2014, 47, 3582

33 K. Nishi, K. Fujii, U.-I. Chung, M. Shibayama and T. Sakai, Phys. Rev. Lett., $2017,119,1$

34 T. Katashima, M. Asai, K. Urayama, U.-I. Chung and T. Sakai, J. Chem. Phys., $2014,140,074902$

35 T. Nakajima, H. Sato, Y. Zhao, S. Kawahara, T. Kurokawa, K. Sugahara and J. P. Gong, Adv. Funct. Mater., 2012, 22, 4426

36 T. Nakajima, Y. Fukuda, T. Kurokawa, T. Sakai, U.-I. Chung and J. P. Gong, ACS Macro Lett., 2013, 2, 518

T. Matsuda, T. Nakajima, Y. Fukuda, W. Hong, T. Sakai, T. Kurokawa, U.-I. 
Chung and J. P. Gong, Macromolecules, 2016, 49, 1865

38 K. Sato, T. Nakajima, T. Hisamatsu, T. Nonoyama, T. Kurokawa and J. P. Gong, Adv. Mater., 2015, 27, 6990

39 Y. Hu, X. Zhao, J. J. Vlassak and Z. Suo, Appl. Phys. Lett., 2010, 96, 121904.

40 C. T. McKee, J. A. Last, P. Russell and C. J. Murphy, Tissue Eng. Part B Rev., $2011,17,155$

41 D. C. Lin, D. I. Shreiber, E. K. Dimitriadis and F. Horkay, Biomech. Model. Mechanobiol., 2009, 8, 345

42 S. V. Panyukov, Sov. Phys. JETP, 1990, 71, 372

43 R. Jedynak, Rheologica Acta, 2015, 54, 29

44 J. F. Marko and E. D. Siggia, Macromolecules, 1995, 28, 8759.

45 F. Kienberger, V. P. Pastushenko, G. Kada, H. J. Gruber, C. Riener, H. Schindler and P. Hinterdorfer, Single Mol., 2000, 1, 123

46 F. Oesterhelt, M. Rief and H. E. Gaub, New J. Phys., 1999, 1, 1

47 R. Begum and H. Matsuura, J. Chem. Soc. Faraday Trans., 1997, 93, 3839

48 E. M. Arruda and M. C. Boyce, J. Mech. Phys. Solids, 1993, 41, 389

49 L. R. G. Treloar, Rep. Prog. Phys., 1973, 36, 755 